\title{
Ueber die Halphen'sche Reaktion und ihren Werth für Butteruntersuchungen.
}

\author{
Von \\ Dr. B. Sjollema und Dr. J. E. Tulleken.
}

Mittheilung aus der Landwirthschaftlichen Reichs-Versuchsstation Groningen (Holland).

Bekanntlich wird bei der Margarinefabrikation oft Baumwollsamenol verwendet; es liegt daher nahe bei der Untersuchung von Butter auf fremde Fette auf dieses Oel zu prüfen. Die beste Reaktion, nämlich die ron $\mathrm{Halph}_{\mathbf{p}}$, tritt aber auch bei Butter auf, welche aus Milch von Kühen, die mit Baumarollsamenmehl gefüttert worden sind, hergestellt ist.

Bei unseren Versuchen, durch welche, wir ermitteln wollten, wie stark die Halphen'sche Reaktion bei derartiger Butter auftreten kann, fiel es uns auf, dass die Farbe der $\mathrm{Hal}_{\mathrm{l}}$ hen'schen Reaktion beim Baumwollsamenöl und bei Margarine anders ausfiel, als bei Butter von Kühen, die mit Baumwollaamenmehl gefüttert waren; bei letzterer war die Farbe mehr purpurroth statt rubinroth. Dies veranlasste uns zu untersuchen, ob vielleicht die Farbe der Halphen'schen Reaktion beim Baumwollsamenöl von zwei oder mehreren Farbstoffen hervorgerufen wird, ron denen einer bei Baumwollsamenmehl-Fütterang nicht in die Milch übergeht und somit auch nicht in der Butter auftritt.

Wir beabsichtigten diese Frage sowohl spektroskopisch wie chemisch zu untersuchen.

Die Ergebnisse unserer spektroskopischen Untersuchungen waren aber derart, dass die chemische Untersuchung alsbald aufgegeben wurde.

Die zu unseren Untersuchungen verwendete Butter wurde erhalten durch Verbuttern der Milch von zwei Kühen, die für den Tag und Kopf mit $3^{1 / 2} \mathbf{~ k g}$ Baumwollsamenmehl, enthaltend $12,3 \%$ Fett, gefüttert wurden und nur wenig Milch gaben, nämlich zusammen ungefähr 14 Liter im Tage.

Zuerst sei mitgetheilt, dass die $\mathrm{Halph}$ en'sche Reaktion sehr stark bei dieser Butter auftrat; ungefähr halb so stark wie sie oft von uns bei Margarine, bei deren Herstellung Baumwollsamenöl verwendet war, beobachtet wurde.

In einem besonderen Falle, wie er bei unserer Butter vorlag, kann also die $\mathrm{Halphen}$ 'sche Reaktion so stark in unverfälschter Butter auftreten, dass da, wo man einen solchen besonderen Fall nicht für ausgeschlossen halten muss, die $\mathrm{Halphen}$ 'sche Reaktion in der Regel ein Beweis für die Anwesenheit fremder Fette in Butter nicht liefern kann, wenn die Reichert-Meissl'sche Zathl keine sichere Auskunft giebt.

Unter normalen Verhältnissen aber ist die $\mathrm{Halphen'sche} \mathrm{Reaktion} \mathrm{bei}$ Butter, welche von einer Baumwollsamenmehl-Fütterung herrührt, nur sehr schwach $^{1}$ ). Dies zeigten unsere Versuche mit Butter von Kühen, die täglich $2 \mathrm{~kg}$ Baumwollsamenmehl erhielten und wenigstens 10 Liter Milch gaben.

1) Es scheint uns nicht angebracht, die Stärke der Reaktion in Procentzahlen auszu- 
Zur Untersuchung der Absorptionsspektra der bei der Halphen'schen Reaktion auftretenden Flüssigkeiten wurde die Butter benutzt, welche von den täglich mit $3^{1 / 2} \mathrm{~kg}$ Baumwollsamenmebl gefütterten Kühen herrührte. Das bei dieser Butter erhaltene Spektrum (bei A uer-Glühlicht) wurde verglichen mit dem Spektrum von Baumwollsamenöl. Es wurden drei Sorten Baumwollsamenöl verwendet, die aber keine Unterschiede in den Absorptionsbändern zeigten. Unsere ersten Beobachtungen ergaben, dass bei der Butter nur ein Absorptionsband von $\lambda$ 506-476 mit einem Absorptions-Maximum ungefähr bei $\lambda 490$, also auf der Grenze von grün und blan auftrat, während das Baumwollsamenöl zwei Bänder lieferte, von denen das eine identisch mit dem der Butter, das andere aber von $\lambda 565-535$ mit einem Absorptions-Maximum ungefähr bei $\lambda 550$, also im Gelben, auftrat. Dieses Ergebniss liess erwarten, dass es möglich sein würde, die Halphen'sche Reaktion von Butter und Baumwollsamenöl spektroskopisch zu unterscheiden. Es zeigte sich aber bei unseren weiteren Untersuchungen, dass das Auftreten der Absorptionsbänder von der Temperatur und von der Dauer des Erhitzens abhängt.

Es wurden deshalb bei unseren weiteren spektroskopischen Untersuchungen die Reaktionen immer genau unter denselben Temperaturverhältnissen gehalten und die 'Temperatur stets gemessen. Damit die Erhitzung längere Zeit fortgesetzt werden konnte, ohne dass der Schwefelkohlenstoff sich verflüchtigte, wurden, besonders bei Erhitzung auf höhere Temperaturen, die Reaktionen in Druckfläschchen ausgeführt. Für Temperaturen bis $90^{\circ}$ wurde in einem Wasserbade, für höhere Temperaturen in einem Oelbade erhitzt. Die niedrigste Temperatur, auf die erhitzt wurde, war $5 \tilde{5}^{\circ}$. Sobald die Farbe genügend stark war, wurde das Erwärmen unterbrochen und bei einem Theile der Flüssigkeit das Absorptionsspektrum untersucht und der andere Theil weiter erhitzt. Zur Ausführung der Reaktion wurden immer gleiche Volumen filtrirtes Butterfett, (bezw. Baumwollsamenöl), Amylalkohol und 1\% Schwefel enthaltender Schwefelkohlenstoff verwendet.

Die folgenden Beobachtungen wurden mehrfach gemacht. Bei Erhitzung auf $55^{\circ} \mathrm{C}$. gab das Baumwollsamenöl nach 4 Stunden ein Absorptionsband im Gelben von $\lambda 565$ bis 535 mit einem Absorptions-Maximum von ungefähr $\lambda 550$. Dasselbe Absorptionsspektrum trat bei der Butter auf, aber erst nachdem längere Zeit erhitzt worden war. Bei längerem Erhitzen des Baumwollsamenöles auf $55^{\circ}$ erschien auch das zweite Band von $\lambda 506$ bis 476 , dagegen schien die Dauer der Erhitzung für die Butter nicht genügend, um dieses zweite Band zum Vorschein zu bringen.

Es wurden dann beide Proben während sieben Stunden auf $65^{\circ}$ erwärmt. Auch bei der Butter traten nun beide Bänder auf (Maximum des einen Bandes bei $\lambda 550$, des anderen bei $\lambda 490$ ). Die beiden Bänder hatten ungefähr dieselbe

drücken, denn bei Baumwollsamenölproben verschiedener Herkunft tritt die Reaktion ebenfalls ungleich stark auf. 
Stärke. Bei dem Baumwollsamenöl. hatte aber die Stärke der Farbe so sehr zugenommen, dass nur das Band mit dem Maximum $\lambda \mathbf{5 5 0}$ wahrzunehmen war. Merkwürdiger Weise war aber das Band bei Durchsicht durch eine dicke Schicht nach links verschoben bis $\lambda 590-564$ mit dem Maximum bei $\lambda 577$.

Die Erhitzung wurde nun fortgesetzt unter Erböhung der Temperatur auf 85 und $100^{\circ}$; für jede Temperatur während ungefähr zwei Stunden. Bei der Butter wurde das Band $\lambda 490$ allmällich stärker, als das Band $\lambda$ 550. Wurde aber das Absorptionsspektrum einer dicken Schicht beobachtet, so war das Band $\lambda 490$ wegen Verdunkelung der rechten Hälfte des Spektrums nicht als ein einzelnes Band zu beobachten. Das Band im Gelben war dann nach links verschoben (bis $\lambda$ 577), wie beim Baumwollsamenöl.

Schliesslich wurde noch während drei Stunden auf $110^{\circ}$ erhitzt. Die Spektra von Butter und Baumwollsamenöi waren auch dann noch gleich. In starker Koncentration und bei breiter Spaltöffnung trat nur das Maximum $\lambda 577$ als ein scharfes Band auf; bei Verdïnnung mit Amylalkohol war es verschoben

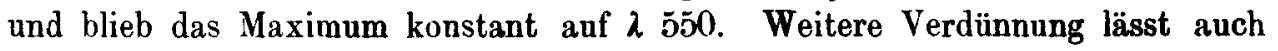
das Band im Blauen (Maximum $\lambda$ 490) als ein einzelnes Band erkennen. Bei noch stärkerer Verdünnung mit Amylalkohol verschwindet das Band $\lambda \mathbf{5 5 0}$ und bleibt nur das Band $\lambda 490$.

Als Ergebniss dieser Untersuchungen haben wir also zu verzeichnen:

1. Die bei der Halphen'schen Reaktion auftretenden Farbstoffe sind die nämlichen für Baumwollsamenöl und für Butter, welche bei Fütterung init Baumwollsamenmehl gewonnen wird.

2. Bei Erhitzung auf niedrige Temperaturen (wie $55^{\circ}$ ) entsteht zuerst ein Absorptionsband im Gelben (Maximum bei $\lambda$ 550), welches bei Beobachtung dicker Schichten nach links verschoben wird (Maximum bei $\lambda$ 570). Bei längerem Erhitzen oder beim Erhöhen der Temperatur tritt ein zweites Band (Maximum bei $\lambda$ 490) auf, das allmählich an Stärke zunimmt, dann aber bei Beobachtung dicker Schichten ohne Verrdünnung wegen Verdunkelung der rechten Hälfte des Spektrums nicht mehr wahrzunehmen ist. Bei starker Verdünnung mit Amylalkohol tritt dann das Band mit einem Maximum bei $\lambda \mathbf{4 9 0}$ dontlich auf; das Band im Gelben hat dann zu sehr an Stärke verloren.

\section{Referate.}

\section{Allgemeine Bestandtheile der Nahrungs- and Genussmittel.}

Virgilio Duccesehi: $Z$ ar Kenntniss der aromatischen Gruppe im Eiweiss molekül. -- Beiträge zur chem. Physiol. und Pathol: 1901, l, 339-346.

Seitdem durch E. Schulze das Phenylalanin aus den Eiweisskörpern der Kürbissamen durch Salzsäure-Spaltung gewonnen war, lag es nahe, diese Verbindung auch in thierischen Eiweissstoffen zu suchen. Vor Kurzem hat nun J o c b em (Zeitschr. 